Essay

\section{A Moonshine Dialogue in Mathematical Physics}

\section{Michel Planat}

Institut FEMTO-ST, CNRS, 15 B Avenue des Montboucons, F-25033 Besançon, France;

E-Mail: michel.planat@femto-st.fr; Tel.: +33-363-082-492

Academic Editor: Palle E.T. Jorgensen

Received: 18 June 2015 / Accepted: 30 July 2015 / Published: 14 August 2015

\begin{abstract}
Phys and Math are two colleagues at the University of Saçenbon (Crefan Kingdom), dialoguing about the remarkable efficiency of mathematics for physics. They talk about the notches on the Ishango bone and the various uses of psi in maths and physics; they arrive at dessins d'enfants, moonshine concepts, Rademacher sums and their significance in the quantum world. You should not miss their eccentric proposal of relating Bell's theorem to the Baby Monster group. Their hyperbolic polygons show a considerable singularity/cusp structure that our modern age of computers is able to capture. Henri Poincaré would have been happy to see it.
\end{abstract}

Keywords: moonshine concepts; Monster group; Grothendieck's dessins d'enfants; modular group; quantum contextuality; finite geometries

PACS classifications: 00A09, 81P45, 81P13, 11G32, 20C34, 11F06, 51E12

Yes I'm crazy Also they say I'm lazy But I'll have my days When I do as I please You won't see the woods While you're a tree No you'll never see the woods While you're a tree (Under Moonshine, The Moody Blues [1]).

I would suggest, as a more hopeful-looking idea for getting an improved quantum theory, that one take as basis the theory of functions of a complex variable. This branch of mathematics is of exceptional beauty, and further, the group of transformations in the complex plane, is the same as the Lorentz group governing the space-time of restricted relativity... the working out of which will be a difficult task for the future (Dirac in 1939, delivered on presentation of the James Scott prize [2]). 


\section{Day 1: The Ishango Bone, Psi and Moonshine}

Phys: Did you hear about the mystery of the Ishango bone found in the Congo by Prof. Jean de Heinzelin? You can see it pictured as Figure 1a.

Math: Yes, this is the oldest known mathematical puzzle, it dates back at least 20,000 years. The bone carries groups of notches totaling 60 in left and right columns and 48 in the center column. A friend of mine found a good explanation of the puzzle, the ancient Africans of Ishango happened to use base 12 for counting [3].

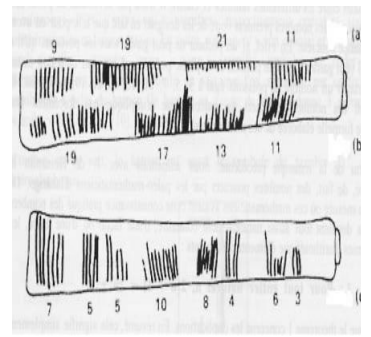

(a)

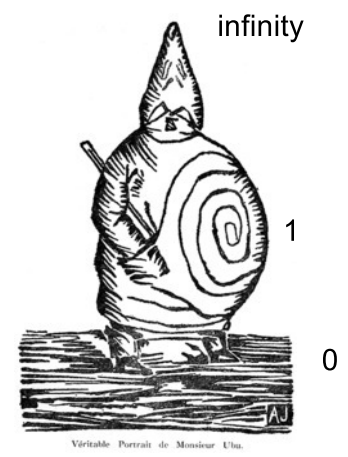

(b)

Figure 1. (a) A schematic of the notches on the Ishango bone; (b) Véritable Portrait de Monsieur Ubu, by Alfred Jarry (1896) [4] (with labeling by the author).

\section{Phys: Why 12 ?}

Math: At that time, Africans used the thumb of a hand to count the bones in the fingers of their hands. Four fingers times three little bones on a hand yield 12 as a counting unit. As there are 24 little bones in the two hands one gets: $60=\sigma(24)$ and $48=\psi(24)$.

Phys: What are these strange symbols $\sigma$ and $\psi$ ? In quantum physics, $\psi(x, t)$ denotes the wave function in space time, but some scientists doubt its reality; it leads to many paradoxes, such as Schrödinger's cat, which may be simultaneously dead or alive. One finally introduced the qubit concept $\psi=a|0\rangle+b|1\rangle$ to model the superposition, but problems arise with two qubits or more with such strange phenomena as non-locality and the non-reality of objects prior to measurements, a phenomenon called contextuality.

Math: In number theory, $\psi(x)$ may designate the second Chebyshev function, a very important concept for looking accurately at the distribution of prime numbers. However, I refer to the Dedekind psi function $\psi(d)=d \prod_{p \mid d}\left(1+\frac{1}{d}\right)$, where the product is over the primes $p$ that divide $d$. At square free numbers $d, \psi(d)$ equals the sum of divisor function $\sigma(d)$. There are papers showing that both functions simultaneously arise for counting the number of maximal commuting sets of a $d$-level quantum system [5].

Phys: You are right. The (general) Pauli group for a qudit (a $d$-level system) is generated by two operators $X$ (shift) and $Z$ (clock) as $\mathcal{P}_{d}=\langle X, Z\rangle$ (generalizing the case of the two-qubit Pauli group when $X$ and $Z$ are the standard Pauli spin matrices). The operators in $\mathcal{P}_{d}$ are organized into $\sigma(d)$ maximal commuting sets (mcs) of size $d-1$, where $\psi(d) \leq \sigma(d)$ of them are "admissible". 
A non-admissible mcs turns out to be transversal to every admissible mcs and can be removed. The geometry of admissible mcs is the projective line $\mathbb{P}_{1}\left(\mathbb{Z}_{d}\right)$, where $\mathbb{Z}_{d}$ is the ring of integers mod $d$, and $\left|\mathbb{P}_{1}\left(\mathbb{Z}_{d}\right)\right|=\psi(d)$. You can easily observe how much different a four-level system is from a two-qubit system. A two-qubit system comprises 15 operators and 15 mcs organized as the smallest thick generalized quadrangle $G Q(2,2)$, while a four-level system has the same number of operators, but only $\sigma(4)=\psi(4)+1=7$ mcs ([5] Table 1$)$.

Math: Both arithmetic functions $\psi(x)$ and $\psi(d)$ can be used to formulate the Riemann hypothesis (RH) about the critical zeros of the Riemann zeta $\zeta(s)$. As for the Dedekind $\psi(n)$, it was first introduced as the index of a particular congruence subgroup $\Gamma_{0}(q)$ of the modular group $\Gamma=\operatorname{PSL}_{2}(\mathbb{Z})$. Congruence subgroups $\Gamma_{0}(p)$, for $p$ prime, are used for defining genus zero surfaces if $p-1$ divides 24 [6]. The relation to RH is as follows. Taking the ratio $R(d)=\psi(d) /(d \log \log d)$, it has been shown that the statement $R(d)>e^{\gamma} / \zeta(2)$ (where $\gamma$ is the Euler constant) is equivalent to RH [7]. As for $\psi(x)$, I know that the logarithmic integral li $[\psi(x)]$ does much better than $\operatorname{li}(x)$ for counting the number of primes. Here, the statement $\operatorname{li}[\psi(x)]-\pi(x)>0$ is equivalent to $\mathrm{RH}[8]$.

Phys: Impressive! I am interested in advanced math for clarifying problems in physics. I like Wolfgang Pauli's quote "There is no God and Dirac is his prophet" [9]. Do you think that God is a mathematician or, like Alfred Jarry, that "God is the tangential point between zero and infinity"? [10]. It is a bit provocative, is it not? Therefore, the number 24 occurs in the "prime groups" $\Gamma_{0}(p)$, as it occurs in the explanation of the tracks in the Ishango bone. It is quite remarkable that a hot topic of physics—quantum entanglement—also relates to 24. The group of automorphisms of Euclidean dense lattices, such as the root lattices $D_{4}$ and $E_{8}$, the Barnes-Wall lattice $\mathrm{BW}_{16}$, the unimodular lattice $D_{12}^{+}$and the Leech lattice $\Lambda_{24}$, may be generated by entangled quantum gates of the corresponding dimension, as shown in [11]. The Leech lattice is the densest known lattice in dimension 24. It has the kissing number 196,560 and automorphism group $\mathbb{Z}_{2} \cdot \mathrm{Co}_{1}$, where the sporadic Conway group $\mathrm{Co}_{1}$ has order about $4.10^{18}$.

Math: However, the story is not finished: the group you mention is a sporadic part of the largest finite group, the Monster group $\mathbb{M}$ of cardinality $|\mathbb{M}|=2^{46} 3^{20} 5^{9} 7^{6} 11^{2} 13^{3} 1719232931414759$ $71 \sim 10^{54}$; this would correspond to the mass in $\mathrm{kg}$ of the known universe. Now, if you add to $\Gamma_{0}(d)(d$ square free) the Fricke involution matrix $\frac{1}{\sqrt{d}}(0,-1 ; d, 0)$, you get another group with a single cusp at $\infty$ called $\Gamma_{0}^{+}(d)$. For prime $d=p$, the group has genus zero if and only if $p$ is in the sequence $\{2,3, \cdots 71\}$ occurring in the factors of $[\mathrm{M} \mid$. This coincidence is again a puzzle when it is rewritten as:

$$
\begin{gathered}
196,884=1+196,883, \\
21,493,760=1+196,883+21,296,876
\end{gathered}
$$

and so on, in which the numbers in the left column occur in the $q$-expansion of the modular $j$-invariant ( $q=e^{2 i \pi \tau}$ and $\tau$ in the upper-half plane), and the numbers at the right column are the sums of dimensions of the smallest irreducible representations of the Monster $\mathbb{M}$ [12]. The coincidence is known as the "monstrous moonshine" (in the sense of being a crazy idea). I quote the Fields medalist Richard Borcherds after his proof of the puzzle with the help of string theoretical concepts: "I sometimes wonder if this is the feeling you get when you take certain drugs. I don't actually know, as I have not tested this theory of mine" [13]. This is an illustration of the pre-established harmony between math and physics. 
Concerning Jarry's quote, I have put the labels 0,1 and $\infty$ on Ubu's portrait in Figure $1 \mathrm{~b}$ in order to illustrate a salient feature of Grothendieck's "dessins d'enfants" [14], another way to approach the moonshine subject.

Phys: Thanks, may be this approach helps to clarify the $\psi$-quantum puzzle. Let us discuss this point tomorrow.

\section{Day 2: The $\psi-Q u a n t u m$ Puzzle Revisited}

Phys: Look at what I see as the simplest $\psi$-quantum diagram of all, a square graph (shown in Figure 2a) that is the paragon of two-qubit Bell's theorem about non-locality ([15] Figure 1). Imagine that Alice and Bob are spatially separated and do electron spin measurements (along the orthogonal directions $x$ and $z$ ) with their Stern-Gerlach. The four (two-qubit) operators involved are denoted $s_{i}$, $i=1 . .4$ as shown in my picture (a), where $X=(0,1 ; 1,0)$ and $Z=(1,0 ; 0,-1)$ are the Pauli spin matrices and a notation, such as $I X$, means the tensorial product of the identity matrix $I$ and the Pauli matrix $X$. There is an edge between two vertices if they commute. As the result of a measurement can only be the eigenvalue \pm 1 of a $s_{i}$, one expects to satisfy the inequality:

$$
C=\left|\left\langle s_{1} s_{2}\right\rangle+\left\langle s_{2} s_{3}\right\rangle+\left\langle s_{3} s_{4}\right\rangle-\left\langle s_{4} s_{2}\right\rangle\right| \leq 2
$$

However, the calculations with the operators $s_{i}$ lead to the norm $\|C\|=2 \sqrt{2}$ instead of two, and the experiments confirm this fact. There are many choices for a square graph with multiple qudits $s_{i}$, and the result is always a maximal violation $2 \sqrt{2}$.

Math: Do you have an explanation of the algebraic equation $C^{2}=8$ ?

Phys: One cannot escape the quantum formalism on this matter. Observe that there is no commuting entangled pair $\left(s_{i}, s_{j}\right)$ in my example; the proof of Bell's theorem does not refer to entanglement. However, I found another argument based on the permutation group $P=\langle\alpha, \beta\rangle$ with the two generators $\alpha=(1,2,4,3)$ and $\beta=(2,3)$; this is pictured in my Figure $2 \mathrm{~b}$ where $i$ means $s_{i}$. Each edge of my drawing corresponds to the same stabilizer subgroup of $P$.

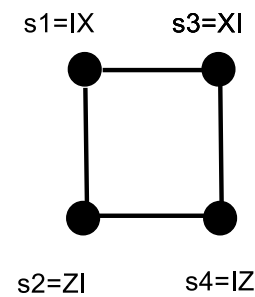

(a)

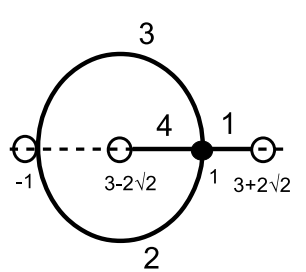

(b)

Figure 2. (a) The square graph of Bell's theorem; (b) the dessin stabilizing the square graph where the label " $i$ " of the edges corresponds to the (operator) vertices " $s_{i}$ " of the square.

Math: Your graph is a Grothendieck "dessin d'enfant", and the generators mean how you go around the black and white vertices, isn't it? And I see that the vertices live in the extension field $\mathbb{Q}(\sqrt{2})$. This means that your dessin can also be seen as a complex algebraic curve over the field of algebraic numbers. Congratulations, this is a nice use of a quite sophisticated mathematical trick. Is your graph (b) the only choice to stabilize the square? 
Phys: You are right, there are essentially four choices, as shown in Figure 1 of [15]. I kept the most asymmetric graph because I have the vague feeling that, as Einstein wrote: "Everything should be made as simple as possible, but not simpler" [16]. The three other choices are too symmetric, I feel one needs some breaking of the symmetry to allow a deeper explanation of Bell's theorem. I suspect that mathematics can help.

Math: You know, I met your dessin before. It corresponds to one (Case I) of the non-normal inclusions of triangle groups classified by David Singerman [17]. The monodromy permutation $P$ that you introduced is the subcover:

$$
X_{0}(4) \rightarrow X_{0}^{+}(2)
$$

of the famous Klein quartic $X(7)=X^{3} Y+Y^{3} Z+Z^{3} X$. The triangle groups corresponding to the modular curves $X_{0}(4)$ and $X_{0}^{+}(2)$ are the congruence subgroup $\Gamma_{0}(4)$ and the smallest moonshine group $\Gamma_{0}^{+}(2)$ that we discussed yesterday. I suspect that your Bell theorem is an elementary stone of a modular physical theory based on the Monster $\mathbb{M}$, a kind of atom. This reminds me of the vortex atoms of Lord Kelvin. The modern language is knot theory, and Witten developed his topological quantum field theory based on this set of ideas.

Even more intriguing, the moonshine group $\Gamma_{0}^{+}(2)$ is related to the Baby Monster group $\mathbb{B}$ of order $2^{41} 3^{13} 5^{6} 7^{2} 11131719233147 \sim 4.10^{33}$ through another puzzling series ([12], p. 20).

$$
\begin{gathered}
a_{1}=4372=1+4371, \quad a_{2}=96,256=1+96,255, \\
a_{3}=1,240,002=2.1+4371+96,255+1,139,374
\end{gathered}
$$

and so on, where the numbers $a_{i}$ correspond to the $q$-expansion of the main modular invariant $T_{2 A}$ (also called a Hauptmodul) for $\Gamma_{0}^{+}(2)$ and the numbers $1,4371,96,255,1,139,374, \ldots$, are all dimensions of the smallest irreducible representations of $\mathbb{B}$. One has the property: $\sum_{i=1}^{24} a_{i}^{2} \bmod (70)=42$ as for the $q$-expansion of $j(q)$ and that of the modular discriminant $\Delta(q)$ [18].

That moonshine for $\mathbb{B}$ related to Bell's theorem is a remarkable coincidence!

Phys: The approach is the application of a simple axiomatic with only two letters $a$ and $b$ [19]. Mathematically, my language is also called a free group $G=\left\langle a, b \mid b^{2}=e\right\rangle$. Elements in the group are words $u$, any combination $u u^{-1}=e$ (the neutral element) is annihilated, and I also write that $b$ is an involution, that is $b^{2}=e$.

The relative size of a subgroup $H$ of $G$ is called the index, which means that there are $n$ inequivalent copies (called cosets) of $H$ that fill up $G$. The action of generators on these cosets creates the permutation group $P$ by the Todd-Coxeter algorithm. You can name the cosets by a word representative; the other elements in the coset are conjugate to the representative. I did it in Figure 3 for the case just discussed where the index is four.

With a little effort, you can check that pairs of cosets on a edge are commuting in the group sense, that is the commutator $(u, v)=u^{-1} v^{-1} u v=e$. In this special case, coset commutation respects quantum commutation.

However, I can show that it is not always the case for a geometry stabilized by a higher index group. Following Gottlob Frege: "Never ask for the meaning of a word in isolation, but only in the context of a sentence" [20]. 


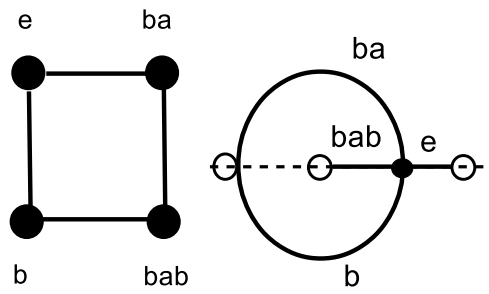

(a)

(b)

Figure 3. (a) The representatives of cosets for the dessin (b) stabilizing the square graph (a). For this special case, mutually-commuting operators correspond to mutually-commuting cosets.

Look at Mermin's pentagram shown on Figure 4b [21]; I labeled the vertices from 1 to 10 and with three-qubit coordinates. The product of operators on a thin line is $I I I$ and $-I I I$ on a thick line, so that, as shown by David Mermin, this pentagram is a contextuality proof $[15,22]$.

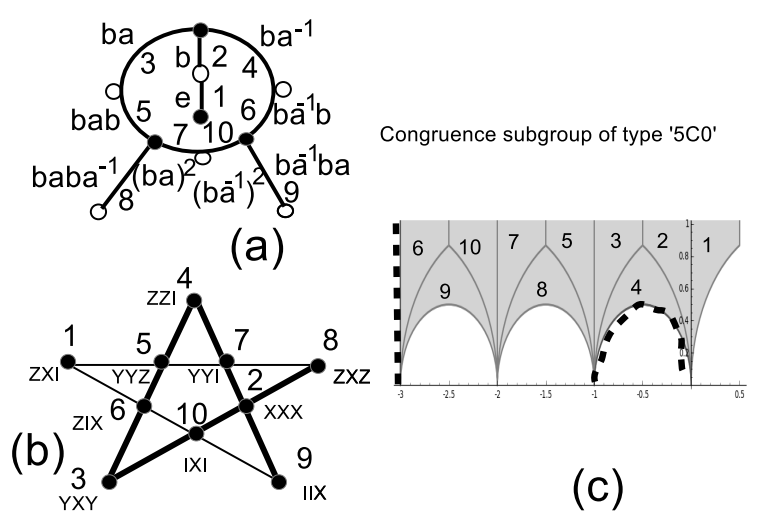

Figure 4. A dessin (a) stabilizing Mermin's pentagram (b) and (c) the fundamental domain of congruence subgroup " $5 C^{0}$ ". The pictures have coordinates in the $G$-set $\{1 . .10\}$ with the corresponding cosets shown in (a) and a set of three-qubit operators shown in (b).

Remember that I was able to stabilize a square with the permutation group (i.e., the dessin d'enfant) shown in Figure $2 \mathrm{~b}$. With the same reasoning, I stabilize the pentagram with a permutation group $P$ generated by the permutations $\alpha=(2,3,4)(5,7,8)(6,9,10)$ and $\beta=(1,2)(3,5)(4,6)(7,10)$, as shown in Figure 4a. Each point on a selected line of the pentagram corresponds to the same stabilizer subgroup $S$ of $P$ (all of the $S$ 's are isomorphic, but they act on different sets of points). Then, in Figure $3 \mathrm{~b}$, I have put the coset representatives for labeling the edges. In this way, you can check that on a thick line of the pentagram, not all cosets are commuting. Of course, the cosets on a (thin) line containing the identity element $e$ are commuting. I call a geometry "contextual" when it happens that at least a line fails to have its points/cosets commuting. In this way, geometric contextuality reflects quantum contextuality [19].

Math: You did not comment on your Figure 4c, but I recognize the tiling of a fundamental domain in the upper-half plane $\mathbb{H}$. I see that the tiles of $\mathbb{H}$ reflect the edge (coset) structure of your dessin, and of course, the tiles also correspond to your three-qubit operators.

Phys: Yes, the "modular" representation of the pentagram is in the spirit of what you explained yesterday. As the generators $\alpha$ and $\beta$ have order three and two, respectively, they build a subgroup $\Gamma^{\prime}$ 
of the modular group $\Gamma$ that is a congruence subgroup of level 5. The set of cusps for $\Gamma^{\prime}$ consists of the $\Gamma^{\prime}$-orbits of $\{\mathbb{Q}\} \cup\{\infty\}$; cusps are at -3 and $\infty$, and they have a width of three. My $\Gamma^{\prime}$ is of type " $5 C^{0}$ " in the Cummins-Pauli classification (http://www.uncg.edu/mat/faculty/pauli/congruence/congruence. html). I used the software Sage to draw the fundamental domain of $\Gamma^{\prime}$ thanks to the Farey symbol methodology. I am not the first to play with Sage on modular aspects of dessins; you can read the essay by Lieven le Bruyn [23].

Math: If your approach makes sense, you should also encounter other moonshine groups, did you?

Phys: It is true that moonshine groups relate to geometric contextuality. There exist six congruence subgroups of the modular group $\Gamma$ that are simultaneously torsion free, of genus zero and index 12 , and three of them have a moonshine group as their normalizer ([24] Table 5). The corresponding dessins can be seen in [25] (Section 2.3.1). Look at the results in my Table 1: the normalizer of $\Gamma(3)$ is $\Gamma$, and three extra congruence subgroups have the normalizer $\Gamma_{0}^{+}(d), d=2,5,6$. It is known that $j^{1 / 3}$ occurs as a generating function for $\Gamma(3)$ ([12], p. 7). I also showed which geometry these groups stabilize.

Tomorrow, I will tell you more about my work inspired by the moonshine topic.

Table 1. Characteristics of torsion-free, genus zero, index 12 congruence subgroups. The notation $K(., \cdots,$.$) means the corresponding complete multipartite graph.$

\begin{tabular}{ccrc}
\hline Group & Normalizer & Geometry & Cusps \\
\hline$\Gamma(3)$ & $\Gamma$ & 11-simplex & $3^{4}$ \\
$\Gamma_{0}(4) \cap \Gamma(2)$ & $\Gamma_{0}^{+}(2)$ & $K(4,4,4)$ & $4^{2} 2^{2}$ \\
$\Gamma_{1}(5)$ & $\Gamma_{0}^{+}(5)$ & 6 -orthoplex & $5^{2} 1^{2}$ \\
$\Gamma_{0}(6)$ & $\Gamma_{0}^{+}(6)$ & $K(6,6)$ & $6^{1} 3^{1} 2^{1} 1^{1}$ \\
$\Gamma_{0}(8)$ & $\cdot$ & $K(4,4,4)$ & $8^{2} 2^{1} 1^{2}$ \\
$\Gamma_{0}(9)$ &. & $K(3,3,3,3)$ & $9^{1} 1^{3}$ \\
\hline
\end{tabular}

\section{Day 3: Dessins d'Enfants, Hyperbolic Polygons, Rademacher Sums}

Phys: It seems not to be widely known that most sporadic groups may be defined as permutation groups $P$ with two generators [26], acting on a $G$-set of cardinality $n$. These representations can be found explicitly in the "Atlas of finite group representations" [27]. As with the previous section, a $P$ may be seen as "dessin d'enfant" $\mathcal{D}$ endowed with a natural topological structure with Euler characteristic $2-2 g=B+W+F-n$ with $B$ black points, $W$ white points, $F$ faces, $n$ edges and genus $g$ (see $[14,15,19]$ for details).

Another remarkable property is that, in many cases, a $P$ (and its $\mathcal{D}$ ) stabilizes a graph/geometry $\mathcal{G}$. First, a pair of elements of the $G$-set attached to $P$ defines a stabilizer subgroup $S$ of $P$. Second, the collection of isomorphic $S$ acting on different subsets of the $G$-set defines the edges/lines of $\mathcal{G}$. A list of the stabilized $\mathcal{G}^{\prime} s$ of a small index can be found in [15] (Tables 1 and 2). In the following, I restrict to "modular dessins" that are defined from a subgroup $\Gamma^{\prime}$ of the modular group $\Gamma$ (as for the pentagram of the previous section). For these $\mathcal{D}$, the valency of black and white points is three and two, respectively (except for the elliptic points, where the valency is one). 
Table 2. Characteristics of a few small "sporadic" fundamental polygons.

\begin{tabular}{cccccccc}
\hline Graph/Geometry & Group & $\boldsymbol{n}$ & $\boldsymbol{g}$ & $\boldsymbol{\nu}_{\mathbf{2}}$ & $\boldsymbol{\nu}_{\mathbf{3}}$ & Cusps & $\boldsymbol{f}$ \\
\hline Hall-Janko & $J_{2}$ & 100 & 0 & 0 & 4 & $1^{2} 7^{14}$ & 33 \\
Livingstone & $J_{1}$ & 266 & 0 & 10 & 5 & $7^{38}$ & 88 \\
McLaughin & $M c L: 2$ & 275 & 10 & 11 & 14 & $11^{1} 22^{12}$ & 88 \\
$T(24)$ & $M_{24}$ & 276 & 10 & 12 & 15 & $23^{12}$ & 12 \\
Ree-Tits GO(2,4) & $\mathrm{T}$ & 1755 & 57 & 91 & 0 & $13^{135}$ & 586 \\
Suzuki & $\mathrm{Suz}$ & 1782 & 70 & 42 & 0 & $1^{1} 3^{137}$ & 595 \\
Janko & $J_{3}$ & 6156 & 321 & 76 & 36 & $19^{324}$ & 2041 \\
Fischer & $\mathrm{Fi}_{23}$ & 31,671 & 1876 & 695 & 27 & $2^{1} 4^{2} 7^{9} 14^{45} 28^{1106}$ & 10,549 \\
Conway & $\mathrm{Co}_{1}$ & 98,280 & 6922 & 0 & 27 & $10^{12} 20^{108} 40^{2400}$ & 32,752 \\
Fischer & $\mathrm{Fi}_{24}^{\prime}$ & 306,936 & 19,409 & 3512 & 0 & $29^{10,584}$ & 155,225 \\
\hline
\end{tabular}

Lieven le Bruyn writes in [23]: "It would be nice to have (a) other Farey-symbols associated to the second Janko group, hopefully showing a pattern that one can extend into an infinite family as in the iguanodon series and (b) to determine Farey-symbols of more sporadic groups".

This is precisely what I did for several "sporadic" iguanodons (dessins d'enfants [14,15]). In Table 2, I list a few of them (those related to $J_{1}, J_{2}, J_{3}, \mathrm{McL}, M_{24}, T, \mathrm{Suz}, \mathrm{Fi}_{23}, \mathrm{Co}_{1}, \mathrm{Fi}_{24}^{\prime}$ ) and give the main characteristics of their fundamental domain $\mathcal{P}$ in the upper-half plane $\mathbb{H}$. All of the groups are represented as non-congruence subgroups of the modular group $\Gamma$. The number $n$ of edges of the dessin $\mathbb{D}$ is the index of the (sporadic) group representation. The genus $g$ of $\mathbb{D}$ equals that of the hyperbolic polygon $\mathcal{P}$; a face of $\mathbb{D}$ corresponds to a cusp of $\mathcal{P}$; the number of black points (respectively of white points) of $\mathbb{D}$ is $B=f+\nu_{3}-1$ (respectively $W=n+2-2 g-B-c$ ), where $f$ is the number of fractions, $c$ is the number of cusps; $\nu_{2}$ and $\nu_{3}$ are the number of elliptic points of order two and three of $\mathcal{P}$, respectively. Elliptic points forbid most of these "sporadic dessins" to be trivalent (when $\left.\nu_{3} \neq 0\right)$ and clean (when $\nu_{2} \neq 0$ ).

For instance, the smallest permutation representation $P$ of our friend, the Conway group $\mathrm{Co}_{1}$, can be found in the Atlas [27]; it may be seen as a dessin $\mathcal{D}$ of index $n=98,280$ (half the kissing number of the Leech lattice). An excellent signature consists of the cycle structure [15,28] about the black and white vertices and the faces of $\mathcal{D}$. For this representation of $\mathrm{Co}_{1}$, one gets the cycles $\left[3^{32,751} 1^{27}, 2^{49140}, 40^{2400} 20^{108} 10^{12}\right][15,28]$. From the cycles, it is straightforward to recognize that there are $32,778=32,751+27$ black points (the first of them have valency three, and the rest consists of elliptic points of order 3, i.e., $\nu_{3}=27$ ), 49,140 white points (with valency two) and $c=2400+108+12=2520$ cusps. The genus $g$ follows from the Euler formula given at the beginning of this section. This $\mathcal{D}$ can also be seen as an hyperbolic polygon $\mathcal{P}$ of the upper-half plane (not shown).

Similarly, the dessin $\mathcal{D}$ for the Tits group with permutation representation of index $n=1755$ has the cycle structure $\left[3^{585}, 2^{8321} 1^{91}, 13^{135}\right]$, and the characteristics are shown in Table 2 . The fundamental domain for this representation of the Tits group $T$ is shown in Figure 5. The geometry $\mathcal{G}$ stabilized by $\mathcal{D}$ is the Ree-Tits octagon $\operatorname{GO}(2,4)$ (see https://en.wikipedia.org/wiki/Generalized_polygon for details about the generalized polygons). 


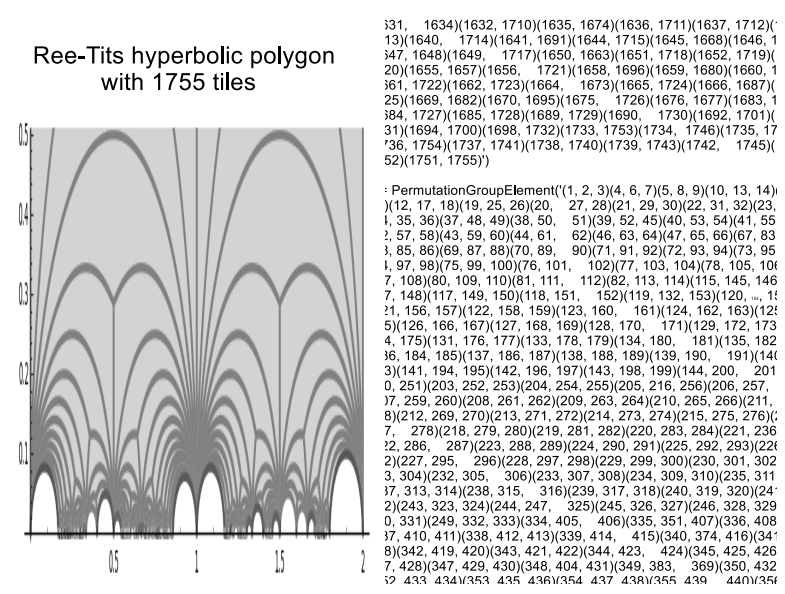

Figure 5. The fundamental domain for the index 1755 permutation group stabilizing the Ree-Tits octagon (details can be found in [27]) and an excerpt of the permutation representation.

Math: Excellent, the next step would be to compute the modular symbols, the relative homology of the extended upper half plane and the corresponding modular forms of weight two and higher. I am curious to see if the non-congruence cusp forms for these "sporadic" polygons have unbounded denominators, as is conjectured [29].

You are close to the "philosophy of cusp forms" of Harish-Chandra. He was studying under Dirac himself, but turned to mathematics when he learned in Princeton that "not every function is analytic" [30]. The keyword for the link between moonshine and the Langlands program is VOA (for vertex operator algebra). The Monster vertex algebra is conjectured (by Edward Frenkel and collaborators) to be the unique holomorphic VOA with charge 24 and partition function $j-744$ (where $j$ is again the modular invariant).

I quote Edward Frenkel [31]: "Mathematics is not about studying boring and useless equations: It is about accessing a new way of thinking and understanding reality at a deeper level. It endows us with an extra sense and enables humanity to keep pushing the boundaries of the unknown."

I suggest that you look at a promising line of moonshine research by Igor Frenkel ("not Edward Frenkel") and John Dunkan [32] based on the use of a Rademacher sum for the modular invariant $j(\tau)=$ $q^{-1}+744+196,884 q+21,493,760 q^{2}+\cdots$, with $q=e^{2 i \pi \tau}$ as:

$$
\begin{gathered}
j(\tau)+12=e^{-2 i \pi \tau}+\lim _{K \rightarrow \infty} \sum_{\substack{0<c<K,-K^{2}<d<K^{2} \\
(c, d)=1}} e^{-2 i \pi \frac{a \tau+b}{c \tau+d}}-e^{-2 i \pi \frac{a}{c}} \\
c
\end{gathered}
$$

with $a, b$ relative integers and $a d-b c=1, \tau \in \mathbb{H}$.

The authors show that the McKay-Thomson series $T_{g}(\tau)$ of an element $g \in \mathbb{M}$ coincides with a generalized Rademacher sum. They expect to get a version of the simplest chiral $3 d$ quantum gravity with 24 charges, as the original construction of the chiral $2 d$ conformal field theory by McKay based on the partition function $j(q)$.

Phys: I am familiar with Rademacher's work. Some time ago, I investigated the thermodynamics of the Euler gas whose partition function is that of the number of unrestricted partitions $p(n)$. This 
was useful to model the low frequency fluctuations (of the $1 / f$ type) occurring in a gas of bosons, like the phonons in a quartz crystal resonator [33]. The mathematics involves the Hardy-Ramanujan circle method in analytic number theory, and this was improved by Rademacher.

Math: Do you think that our mathematics is the real world?

Phys: As a provisional response, I offer you a quote of Stephen Hawking from his lecture "Godel and the end of the universe" [34]: "In the standard positivist approach to the philosophy of science, physical theories live rent free in a Platonic heaven of ideal mathematical models... But we are not angels, who view the universe from the outside. Instead, we and our models are both part of the universe we are describing. Thus a physical theory is self referencing, like in Godel's theorem. One might therefore expect it to be either inconsistent or incomplete. The theories we have so far are both inconsistent and incomplete."

Math: However, you should know Dyson's words in 1981: "I have a sneaking hope, a hope unsupported by any facts or any evidence, that sometime in the twenty-first century physicists will stumble upon the Monster group, built in some unsuspected way into the structure of the universe. This is of course only a wild speculation, almost certainly wrong" [35]. Today, we also know that monstrous moonshine has a shadow, umbral moonshine [36], that is potentially of huge interest for theoretical physics.

\section{Conflicts of Interest}

The author declares no conflict of interest.

\section{References}

1. The Moody Blues, Octave 02 under Moonshine, 1978. Available online: https://www.youtube.com (accessed on 1 March 2015).

2. Dirac, P.A.M. The Relation between Mathematics and Physics. Proc. Roy. Soc. (Edinburgh) II 1938-1939, 59, 122-129.

3. Pletser, V.; Huylebrouck, D. The Ishango artefact: The missing base 12 link. Forma 1999, 14, 339-346.

4. Jarry, A. Ubu Roi, in Oeuves Complètes I; Gallimard, Bibliothèque de la Pléiade: Paris, France, 1972.

5. Planat, M. Pauli graphs when the Hilbert space dimension contains a square: Why the Dedekind psi function? J. Phys. A Math. Theor. 2011, 44, doi:10.1088/1751-8113/44/4/045301.

6. Shimura, G. Introduction to the Arithmetic Theory of Automorphic Functions; Princeton Press: Princeton, NJ, USA, 1971.

7. Solé, P.; Planat, M. Extreme values of the Dedekind $\psi$ function. J. Comb. Numb. Theory 2011, 3, 166.

8. Planat, M.; Solé, P. Improving Riemann prime counting. Notes Number Theory Disc. Math. 2015, 21,1 . 
9. Heisenberg, W. Physics and Beyond; Encounters and Conversations; Harper \& Row: New York, NY, USA, 1971. Available online: http://en.wikiquote.org/wiki/Wolfgang_Pauli (accessed on 1 March 2015).

10. Jarry, A. Exploits and Opinions of Doctor Faustroll, Pataphysician; Taylor, S.W., Translator; Exact Change: Boston, MA, USA, 1996; p. 114. Available online: http://72.52.202.216/ fenderse/Dr_ Faustroll.pdf (accessed on 18 June 2015).

11. Planat, M. Multipartite entanglement arising from dense Euclidean lattices in dimensions 4-24. Phys. Scr. 2012, 147, doi:10.1088/0031-8949/2012/T147/014025.

12. Gannon, T. Monstrous moonshine: The fist twenty-five years. Bull. Lond. Math. Soc. 2005, 38, 1.

13. Singh, S. Interview with Richard Borcherds, The Guardian (28 August 1998). Available online: http://simonsingh.net/media/articles/maths-and-science/interview-with-richard-borcherds/ (accessed on 1 March 2015).

14. Grothendieck, A. Geometric Galois Actions: 1. Around Grothendieck's Esquisse d'un Programme; Schneps, L., Lochak, P., Eds.; Cambridge University Press: Cambridge, UK, 1997.

15. Planat, M.; Giorgetti, A.; Holweck, F.; Saniga, M. Quantum contextual finite geometries from dessins d'enfants. Int. J. Geom. Meth. Mod. Phys. 2015, 12, doi:10.1142/S021988781550067X.

16. A Quote Attributed to Einstein, the Quote Investigator (Exploring the Origins of Quotations). Available online: http://quoteinvestigator.com/2011/05/13/einstein-simple/\#more-2363 (accessed on 1 March 2015).

17. Hoshino, K. The Belyi functions and dessins d'enfants corresponding to the non-normal inclusions of triangle groups. Math. J. Okayama Univ. 2010, 52, 45-60.

18. He, Y.; McKay, J. Moonshine and the Meaning of Life; Cornell University Library: Ithaca, NY, USA, 2014.

19. Planat, M. Geometry of contextuality from Grothendieck's coset space. Quant. Inf. Proc. 2015, $14,2563-2575$.

20. Frege, G. Die Grundlagen der Arithmetik: Eine Logisch-Mathema-Tische Untersuchung über den Begriff der Zahl; W. Koebner: Breslau, Poland, 1884; Reprint in G. Olms: Hildesheim, Germany, 1961.

21. Mermin, N.D. Hidden variables and two theorems of John Bell. Rev. Mod. Phys. 1993, 65, 803-815.

22. Planat, M. It from qubit: How to draw quantum contextuality. Information 2014, 5, 209-218.

23. Lieven, L. Monsters and Moonshine. Available online: http://win.ua.ac.be/ lebruyn/b2hd-LeBruyn 2012a.html (accessed on 1 March 2015).

24. Sebbar, A. Modular subgroups, forms, curves and surfaces. Canad. Math. Bull. 2002, 45, 294-308.

25. He, Y.H.; McKay, J.; Read, J. Modular subgroups, dessins d'enfants and elliptic $K_{3}$ surfaces. LMS J. Comput. Math. 2013, 16, 271-318.

26. Mazurov, V.D. Minimal Permutation Representation of Thompson's Simple Group; Translated from Algebra i Logika; Plenum Publishing Corporation: Berlin, Germany; Heidelberg, Germany, 1989; Volume 27, pp. 562-580. 
27. Wilson R.; Walsh P.; Tripp J.; Suleiman I.; Parker R.; Norton S.; Nickerson S.; Linton S.; Bray J.; Abbott R. ATLAS of Finite Group Representations-Version 3. Available online: http://brauer.maths.qmul.ac.uk/Atlas/v3/ (accessed on 1 March 2015).

28. Lando, S.K.; Zvonkin, A.K. Graphs on Surfaces and Their Applications; Springer Verlag: Berlin, Germany, 2004.

29. Li, W.; Long, L. Atkin and Swinnerton-Dyer Congruences and Noncongruence Modular Forms; Cornell University Library: Ithaca, NY, USA, 2013.

30. Howe, R. A Biographical Memoir; National Academy of sciences: Washington, DC, USA, 2011.

31. Frenkel, E. The Atlantic, 9 October 2013. Available online: http://www.theatlantic.com/technology/ archive/2013/10/the-nobel-prize-in-physics-is-really-a-nobel-prize-in-math/280430/ (accessed on 1 March 2015).

32. Duncan, J.F.R.; Frenkel, I.B. Rademacher sums, moonshine and gravity. Commun. Number Theory Phys. 2009, 5, 1-128.

33. Planat, M. Quantum $1 / f$ noise in equilibrium: From Planck to Ramanujan. Physica A 2003, 318, 371, doi:10.1016/S0378-4371(02)01659-X.

34. Hawking, S. Godel and the End of the Universe, a Lecture. Available online: http://www.hawking. org.uk/godel-and-the-end-of-physics.html (accessed on 1 March 2015).

35. Roberts, S. Curiosities: Pursuing the Monster, Institute of Advanced Study (Fall 2013 Issue). Available online: https://www.ias.edu/ias-letter/roberts-monster (accessed on 1 March 2015).

36. Duncan, J.F.R.; Griffin, M.J.; Ono, K. Proof of the Umbral Moonshine Conjecture; Cornell University Library: Ithaca, NY, USA, 2015.

(c) 2015 by the author; licensee MDPI, Basel, Switzerland. This article is an open access article distributed under the terms and conditions of the Creative Commons Attribution license (http://creativecommons.org/licenses/by/4.0/). 